\title{
Editorial Note: Molecular Imaging and Mechanobiology
}

This special edition of Cellular and Molecular Bioengineering focuses on the intersection of molecular imaging and mechanobiology. Despite the fact that mechanobiology research is rapidly maturing, there remain the vexing questions of where and when mechanosensation occurs, and what is its exact chemical identity. The importance of answering these questions is underscored by the fact that mechanobiology is now widely recognized to underlie atherosclerosis, cancer, developmental biology, bone remodeling, and numerous other physiological and pathophysiological phenomena. In addition, how mechanical environmental cues are perceived by cells, transformed into molecular signals, and coordinated into mechanobiological responses remains an extraordinarily difficult problem to solve because of the complex temporal and spatial control of signaling performed by cells. Nevertheless, major advances in molecular imaging, coupled with bioengineering approaches, are now well poised to uncover the molecular origins of mechanosensation and the attendant processes of mechanobiology. We hope that this assembly of papers from experts in the integration of imaging and mechanobiology will help readers understand existing cutting-edge research and provide inspiration for the development of new avenues for research in mechanobiology.

We start with papers describing studies that exploit the sensitivity of fluorescence molecules to their surroundings. In a review by Qiu et al. the authors highlight recent developments in single molecule detection including smFRET (single molecule Förster resonance energy transfer), PIFE (protein induced fluorescence enhancement), magnetic tweezers and Slimfield (high-resolution cell imaging microscopy). These experimental platforms demonstrate the power of fluorescence to assess the activity of individual molecules in space and time, tools that could form the basis of understanding the role of forces in modulating cellular transcription and translation. Seong et al. further provide an overview of fluorescence technologies, particularly fluorescence resonance energy transfer (FRET), and the potential of FRET-based molecular biosensors for the visualization of enzymatic activities of Src, and focal adhesion kinase (FAK) at subcellular compartments in live cells. Using FRET technology, Meng et al. report new biosensors incorporated into actinin, filamin, and spectrin in endothelial cells (ECs) and into collagen-19 in C. elegans.
These biosensors were applied to live cells to reveal a higher intracellular mechanical stress at the leading edge of a migrating cell. External mechanical shear stress was also shown to cause a gradient of stress distribution at subcellular levels in ECs. Transgenic $C$. elegans labeled with a collagen-based FRET biosensor further revealed tissue stress distributions in a living organism. Additional subcellular investigations are reported by Nishio et al. who used FRET-based biosensors to demonstrate that cell orientation before shear stress application can significantly affect RhoA and Racl responses to mechanical stimulation. When vascular ECs initially oriented parallel to the direction of flow were exposed to shear stress, RhoA and Rac1 activities were activated primarily in the upstream and the downstream regions of cells, respectively. In contrast, RhoA, but not Rac1, activities were induced by shear stress at the upstream regions in ECs pre-oriented perpendicular to the flow. Consistent with uncovering temporal and molecular length scale insight into mechanosensation, Tabouillot and colleagues used time-correlated single photon counting (TCSPC) to measure fluorescence lifetimes, and performed fluorescence correlation spectroscopy on lipoid probes intercalated selectively into liquid ordered (e.g. membrane rafts) and liquid disordered phases of the plasma membrane of endothelial cells. Analysis of fluorescence lifetime and diffusion coefficients revealed that the membrane rafts and non raft portions of the membrane were differentially sensitive to fluid shear stress, thus uncovering a new understanding of spatial control of mechanosensation. In summary, these studies represent how single cell imaging of reporters with molecular-scale sensitivity can elucidate cellular behavior not attainable with more traditional assays that depend on large cell sample sizes, cell lysing, and large protein concentrations. These single cell/single molecule assays are especially important when considering that the health and dysfunction of many cells are related to the high temporal and spatial gradients in force found in vivo.

Fluorescence microscopy was combined with computational analysis methods to advance our understanding of the dynamics of cellular substructures in live cells based on analysis of imaging data. For example, Kaunas and Deguchi review the role of myosin II in the sensing of forces by stress fibers in cells under cyclic stretch. A mathematical model was 
proposed to provide mechanistic insights on the role of myosin II in transmitting external strain into intracellular stress fiber tension thus demonstrating that computational methods must be tightly integrated with image analysis to uncover mechanosensing phenomena. Demchouk et al. developed a method for tracking the tips of microtubules (MTs) obtained by digital fluorescence imaging microscopy. This method can estimate the filament tip structures with high precision, typically at nanometer levels. The results revealed that the tips of MTs within live cells are not all blunt, but highly variable. Looking at actin, Huang et al. designed a quantitative image analysis strategy to measure the spatiotemporal distribution of actin edge ruffling. This strategy has led to the successful detection of a rapid and nondirectional increase in edge ruffling of ECs upon serum stimulation. Such studies should enable future work on the underlying mechanisms and dynamics of cell polarization in response to internal and external mechanical cues.

Fluorescence microscopy also aided in the elucidation of a mechanics-based mechanism that may underlie endothelial cell dysfunction related to diabetes, a major and growing public health concern. In that study, Solomon et al. revealed that the glycation of collagen can impair EC alignment in response to mechanical stretch. In fact, glycated collagen caused a reduced focal adhesion kinase phosphorylation and localization at focal adhesion sites. EC permeability was also altered by glycated collagen when compared to native collagen. Therefore, it is possible that risk factors for diabetes can couple with mechanobiological mechanisms to result in pathophysiological indications in patients. Imaging technology can also elucidate cellular and molecular behaviors in vivo. With advanced multi-photon microscopy, Liang et al. visualized cell populations in human skin, and studied the biomechanical properties of the stratum corneum and epidermis based on quasi-static mechanical deformation. A 2-D elastic registration algorithm was used to analyze the images before and after deformation to determine displacements in different skin layers. This group showed that a significant difference in elastic properties between the stratum corneum and the rest of the epidermis can be detected using this approach.
In conclusion, we have assembled an array of research studies and reviews focusing on the integration of molecular imaging and mechanotransduction, spanning from single-molecule imaging to tissue levels. These presentations should not only introduce readers to cutting-edge technologies involving molecular imaging but also advance insight into and systematic understanding of mechanotransduction. Equally important, we point out that preparation of this special issue coincided with the first Biomedical Engineering Society (BMES) sub-discipline meeting which was held jointly with the Society for the Physical Regulation of Biology and Medicine (SPRBM) in January of 2011 in Miami Beach, Florida. This meeting was held to chart a strategy for assessing how molecular mechanobiology can address major current health challenges. As outlined in the accompanying white paper and meeting minutes, this strategy includes the integration of mechanobiology principles into K-16 biology education, increasing the recognition by the National Institutes of Health on the promise of integrating biology and mechanics, and the promotion of increased interaction between engineers, physical scientists, life scientists, and clinicians. Such interaction should help the healthcare community to better understand the causes of modern disease, develop new strategies for their detection, and devise new technologies for their intervention and cure.

Peter J. Butler

Department of Bioengineering

The Pennsylvania State University

Electronic mail: pjbbio@engr.psu.edu

YINGXIAO WANG

Department of Bioengineering \& Beckman Institute for Advanced Science and Technology, Department of Integrative and Molecular Physiology, Neuroscience Program Center for Biophysics and Computational Biology, Institute for Genomic Biology University of Illinois

Electronic mail: yingxiao@ad.uiuc.edu 